\title{
EL AUDITOR INTERNO Y SU ROL ANTE EL FRAUDE Y LA CORRUPCIÓN
}

\author{
THE INTERNAL AUDITOR AND ITS ROLE AGAINST FRAUD \\ AND CORRUPTION
}

\section{Jeri GLORIa Ramón RufFNeR*}

Docente Principal de la Facultad de Ciencias Contables - UNMSM

[Recepción: Setiembre de 2011/ Conformidad: Noviembre 2011]

\section{RESUMEN}

El presente artículo se ha basado en el estudio y analisis de los sistemas de control interno que vienen funcionando actualmente en entidades que son responsables de la gobernabilidad del país y la rendición de cuentas de los recursos que hayan administrado.

En el desarrollo determinaremos la calidad del sistema de control interno que se viene aplicando en estas entidades, y si éstas son adecuadas a los requerimientos de gestión moderna, de rendición de cuentas oportunas confiables, ademá si las medidas aseguran la disminución de los niveles de corrupción en el aparato estatal. Todo ello, si los sistemas de control interno aseguran el cumplimiento de su misión, visión, objetivos y metas institucionales para la cual fueron creadas .

Si de la evaluación y análisis de los resultados de la investigación determinamos que estos sistemas de control interno no son capaces de concebir el control integral, propondremos una modelo de sistema de control interno, con su respectiva metodología de implantación que busque aplicar un control de advertencia o de prevención en los diferentes niveles organizacionales de las Entidades Públicas, en especial en aquellas que la ciudadania considera como entidades no confiables.

Palabras Clave: Control Interno, Entidades del Estado, Sector Gubernamental.

\begin{abstract}
This article is based on the study and analysis of internal control systems are currently operating in entities that are responsible for the country's governance and accountability of the resources to be managed.

In the development will determine the quality of internal control system has been implemented in these institutions, and whether they are suitable to the requirements of modern management, accountability timely reliable, Adema if measures ensure the reduction of corruption levels in the state apparatus. All this, if the internal control systems ensure compliance with its mission, vision, objectives and institutional goals for which they were created.

If the assessment and analysis of the results of the investigation determined that these internal control systems are not capable of understanding the integral control, we propose a model of internal control system and the respective implementation methodology that seeks to apply a control warning or prevention at different organizational levels of public entities, especially those that citizenship considered untrusted entities.
\end{abstract}

Keywords: Internal Control, Agencies of the State, Government Sector

* Doctora en Ciencias Contables y Mgister en Contabilidad. Socia Principal d la Sociedad de Auditoria "Ramon Ruffner y Asoc. S.C.” Docente de Postgrado y Pregrado de la Fcaultad de Ciencias Contables - UNMSM. Perito Contable Judicial. Actual Directora Administrataiva de la Fcaultad de Ciencias Contables - UNMSM. 


\section{INTRODUCCIÓN}

El presente artículo hace referencia a sistemas de control interno que vienen funcionando actualmente en las entidades públicas y empresas del Estado, en el marco normativo de la Ley No 27785, Ley Orgánica del Sistema Nacional de Control y de la Contraloría General de la República.

En ese contexto de la realidad peruana, consideramos como antecedentes la investigación y la evolución histórica que ha tenido el estudio y la implantación de los sistemas de control interno en el sector gubernamental; por ello, tomaremos como referencia el marco normativo emitido en éste sector, que es la siguiente:

La Organización Internacional de Entidades Fiscalizadoras Superiores- INTOSAI, fundada en 1953, y que reúne entre sus miembros a más de 170 Entidades Fiscalizadoras Superiores (EFS), entre ellas la Contraloría General de la República de Perú (CGR), aprobó en 1992 las "Directrices para las normas de control interno"

El Informe COSO (Comité de Organismos Patrocinadores) incorporó en una sola estructura conceptual los distintos enfoques existentes en el ámbito mundial y actualizó los procesos de diseño, implantación y evaluación del control interno

En setiembre de 2004, el COSO emite el documento "Gestión de Riesgos Corporativos - Marco Integrado", promoviendo un enfoque amplio e integral en empresas y organizaciones gubernamentales

En el XVIII INCOSAI, realizado el 2004 en Budapest, se aprobó la "Guía para las normas de control interno del sector público"

\section{EL CONTROL INTERNO}

La causa principal que dio origen al Control Interno fue la "gran empresa" A finales de este siglo, como consecuencia del notable aumento de la producción, los propietarios de los negocios se vieron imposibilitados de continuar atendiendo personalmente los problemas productivos, comerciales y administrativos, viéndose forzados a delegar funciones dentro de la organización conjuntamente con la creación de sistemas y procedimientos que previeran o disminuyeran fraudes o errores, debido a esto comenzó a hacerse sentir la necesidad de llevar a cabo un control sobre la gestión de los negocios, ya que se había prestado más atención a la fase de producción y comercialización que a la fase administrativa u organizativa, reconociéndose la necesidad de crear e implementar sistemas de control como consecuencia del importante crecimiento operado dentro de las entidades; el desarrollo industrial y económico de los negocios, propició una mayor complejidad en las entidades y en su administración, surgiendo la imperiosa necesidad de establecer mecanismos, normas y procedimientos de control que dieran respuesta a las nuevas situaciones.

Los contadores idearon la "comprobación interna” para asegurarse contra posibles errores y fraudes. "La comprobación interna es el término con el que se llamaba a lo que es hoy Control Interno, que era conocida como la organización y coordinación del sistema de contabilidad y los procedimientos adoptados; que tienen como finalidad brindar a la administración, hasta donde sea posible y práctico, el máximo de protección, control e información verídica". El término de Control Interno reemplazó al de comprobación interna, debido a un cambio conceptual, ya 
que el contenido del mismo ha sufrido una considerable evolución.

En el mundo se le ha prestado una gran atención a la necesidad de elevar las exigencias en los Controles Internos, a partir de la década del 70, por el descubrimiento de muchos pagos ilegales, malversaciones y otras prácticas delictivas en los negocios, por lo que terceras personas quisieron conocer por qué los Controles Internos de las entidades no habían prevenido a la administración de la ocurrencia de esas ilegalidades. El Control Interno ha sido preocupación de la mayoría de las entidades, aunque con diferentes enfoques y terminologías, lo cual se puede evidenciar al consultar los libros de texto de auditoría, los artículos publicados por organizaciones profesionales, universidades y autores individuales; generalmente el Control Interno se ha considerado como un tema reservado solamente para los contadores.

El amplio conjunto de actividades que son necesarias conocer y dominar en la dirección de una empresa, ocupa un lugar importante el Control Interno, el cual reúne los requerimientos fundamentales de todas las especialidades contables, financieras y administrativas de una entidad. El propósito básico del Control Interno es el de promover la operación eficiente de la organización. Está formado por todas las medidas que se toman para suministrar a la administración la seguridad de que todo está funcionando como debe. Los Controles Internos se implantan para mantener la entidad en la dirección de sus objetivos y tiene como principal finalidad la protección de los activos contra pérdidas que se deriven de errores intencionales o no intencionales en el procesamiento de las transacciones y manejo de los activos correspondientes.

\section{EL CONTROL INTERNO Y LA AUDITORIA}

La disciplina de la Auditoría ha estado en constante evolución para tratar de garantizar la fiabilidad de la información económica financiera demandada socialmente. "La auditoría es un proceso sistemático, practicado por los auditores de conformidad con normas y procedimientos técnicos establecidos, consistentes en obtener y evaluar objetivamente las evidencias sobre las afirmaciones contenidas en los actos jurídicos o eventos de carácter técnicos, económicos, administrativos y otros, con el fin de determinar el grado de correspondencia entre las afirmaciones, las disposiciones legales vigentes y los criterios establecidos."

La auditoría es una de las aplicaciones de los principios científicos de la contabilidad, basada en la verificación de los registros patrimoniales de las haciendas para observar su exactitud. Su importancia es reconocida desde los tiempos más remotos.

La auditoría es una función de dirección cuya finalidad es analizar y apreciar, con vistas a las eventuales las acciones correctivas, el control interno de las organizaciones para garantizar la integridad de su patrimonio, la veracidad de su información y el mantenimiento de la eficacia de sus sistemas de gestión; es la actividad por la cual se verifica la corrección contable de las cifras de los estados financieros; es la revisión misma de los registros y fuentes de contabilidad para determinar la racionabilidad de las cifras que muestran los estados financieros emanados de ellos. 


\section{MARCO NORMATIVO DEL CONTROL INTERNO EN EL PERÚ.}

Sin lugar a dudas desde la dación de la Ley No 28716 Ley de Control Interno de las Entidades del Estado de fecha 17.Abr.2006 y las Nomas de Control Interno dictadas por la Contraloría General a través de la Resolución No 320-2006-CG de fecha 03.Nov.2006, elaboradas a la luz del denominado Informe COSO, se dio un cambio revolucionario en el Sector Público, puesto que hasta ese momento la idea del control interno estaba centralizada en el proceso de elaboración de la información financiera, tema sobre el cual los Contadores Públicos llevábamos ventaja, por ser asuntos que correspondían a nuestra formación profesional; al entrar en vigencia estas nuevas normas, las autoridades públicas responsables de la implementación y funcionamiento del control Interno, no supieron que medidas adoptar y buscaron el apoyo de los Contadores Públicos con experiencia en el ejercicio de la Auditoria Gubernamental, no obstante debido a que esta vez se trataba de un sistema multidireccional que se interrelacionaba a través de siete (7) componentes: Ambiente de Control, la Evaluación de Riesgos, Actividades de Control Gerencial, Actividades de Prevención y Monitoreo, Sistemas de Información y Comunicación, Sistema de Resultados y Compromiso de Mejoramiento; que requería una conocimiento de la organización en todas sus dimensiones.

En diciembre de 2007, sucedió un hecho muy importante que condicionaría la implementación del control interno en el conglomerado de instituciones que conforman el Poder Ejecutivo, se trató de la aprobación de "LOPE” - Ley No 29158 - Ley Orgánica del Poder Ejecutivo, la cual estableció los principios y las normas básicas de organización, competencias y funciones del Poder
Ejecutivo, como parte del Gobierno Nacional; las funciones, atribuciones y facultades legales del Presidente de la República y del Consejo de Ministros; las relaciones entre el Poder Ejecutivo y los Gobiernos Regionales y Locales; la naturaleza y los requisitos de creación de Entidades Públicas y los Sistemas Administrativos que orientan la función pública, en el marco de la Constitución Política del Perú y la Ley de Bases de la Descentralización; a la luz de esta norma las entidades públicas presentarían al Congreso de la República sus nuevas propuestas de Ley de Organizaciones y Funciones, sin embargo debido a que la capacidad de atención de este poder era menor a la demanda que había de él, se produjo un tiempo de espera significativo que traería como efecto el impedimento de iniciar el proceso de cambios estructurales requeridos por LOPE.

Es así que desde la dación de LOPE, se advierte que este afecta adversamente a la implementación de Control Interno a la luz de la Ley No 28716 , puesto que si no se contaba con una adecuada estructura organizativa, el componente denominado Ambiente de Control, sobre el cual se inserta el control interno, no tendrá sostenibilidad y por ello sería inviable como sistema.

Con el Decreto de Urgencia No 0672009 del 22.Jun.2009, se condiciona la implementación de la Ley de Control Interno en las entidades públicas, a la aprobación previa de Ley de Organización y Funciones $y$, sus documentos de gestión.

\section{LOS ÓRGANOS DE CONTROL INSTITUCIONAL}

Funciones generales

El Reglamento de los Órganos de Control Institucional, en el artículo $20^{\circ}$.- señala las siguientes funciones del OCI: 
Ejercer el control interno posterior a los actos y operaciones de la entidad sobre la base de los lineamientos y cumplimiento del Plan Anual de Control a que se refiere el artículo $7^{\circ}$ de la Ley, así como el control externo a que se refiere el artículo $8^{\circ}$ de la Ley, por encargo de la Contraloría General.

Efectuar auditorias a los estados financieros y presupuestarios de la entidad, así como a la gestión de la misma, de conformidad con los lineamientos que emita la Contraloría General.

Ejecutar las labores de control a los actos y operaciones de la entidad que disponga la Contraloría General. Cuando estas labores de control sean requeridas por el Titular de la entidad y tengan el carácter de no programadas, su ejecución se efectuará de conformidad con los lineamientos que emita la Contraloría General.

Ejercer el control preventivo en la entidad dentro del marco de lo establecido en las disposiciones emitidas por la Contraloría General, con el propósito de contribuir a la mejora de la gestión, sin que ello comprometa el ejercicio del control posterior.

Remitir los Informes resultantes de sus labores de control tanto a la Contraloría General como al Titular de la entidad y del sector, cuando corresponda, conforme a las disposiciones sobre la materia.

Actuar de oficio cuando en los actos y operaciones de la entidad se adviertan indicios razonables de ilegalidad, de omisión o de incumplimiento, informando al Titular de la entidad para que se adopten las medidas correctivas pertinentes.

Recibir y atender las denuncias que formulen los servidores, funcionarios públicos y ciudadanía en general, sobre actos y operaciones de la entidad, otorgándole el trámite que corresponda a su mérito, conforme a las disposiciones emitidas sobre la materia.
Formular, ejecutar y evaluar el Plan Anual de Control aprobado por la Contraloría General, de acuerdo a los lineamientos y disposiciones emitidas para el efecto.

Efectuar el seguimiento de las medidas correctivas que implemente la entidad como resultado de las labores de control, comprobando y calificando su materialización efectiva, conforme a las disposiciones de la materia.

Apoyar a las Comisiones que designe la Contraloría General para la ejecución de las labores de control en el ámbito de la entidad.

Asimismo, el Jefe del OCI y el personal de dicho Órgano colaborarán, por disposición de la Contraloría General, en otras labores de control, por razones operativas o de especialidad.

Verificar el cumplimiento de las disposiciones legales y normativa interna aplicables a la entidad por parte de las unidades orgánicas y personal de ésta.

Formular y proponer a la entidad, el presupuesto anual del OCI para su aprobación correspondiente.

Cumplir diligente y oportunamente con los encargos y requerimientos que le formule la Contraloría General.

Cautelar que la publicidad de los Informes resultantes de sus acciones de control se realice de conformidad con las disposiciones de la materia.

Cautelar que cualquier modificación del Cuadro para Asignación de Personal, así como de la parte correspondiente del Reglamento de Organización y Funciones, relativos al OCI se realice de conformidad con las disposiciones de la materia.

Promover la capacitación permanente del personal que conforma el OCI, incluida la Jefatura, a través de la Escuela Nacional de Control de la Contraloría General o de cualquier otra Institución universitaria o de nivel 
superior con reconocimiento oficial en temas vinculados con el control gubernamental, la Administración Pública y aquellas materias afines a la gestión de las organizaciones.

Mantener ordenados, custodiados y a disposición de la Contraloría General durante diez (10) años los informes de control, papeles de trabajo, denuncias recibidas y los documentos relativos a la actividad funcional de los OCI, luego de los cuales quedan sujetos a las normas de archivo vigentes para el sector público. El Jefe del OCI adoptará las medidas pertinentes para la cautela y custodia del acervo documental.

Cautelar que el personal del OCI dé cumplimiento a las normas y principios que rigen la conducta, impedimentos, incompatibilidades y prohibiciones de los funcionarios y servidores públicos, de acuerdo a las disposiciones de la materia.

Mantener en reserva la información clasificada obtenida en el ejercicio de sus actividades.

Otras que establezca la Contraloría General.

Plan Anual de Control

El Plan Anual de Control, es aprobado por el Contralor General quien autoriza aprobar los Lineamientos de Política para la formulación de los planes de control de los Órganos del Sistema Nacional de Control año 2009 y la Directiva Nº 012 -2008-CG/ PL "Formulación y Evaluación del Plan Anual de Control de los Órganos de Control Institucional para el año 2009” Publicado 01/11/2008.

\section{A. LINEAMIENTOS DE POLÍTICA GENERAL}

El control preventivo pondrá énfasis en la programación de Veedurías a los procesos de selección en sus distintas modalidades, en las Veedurías a la ejecución de contratos de adquisición de bienes, servicios y obras; y en las Veedurías del Sistema Nacional de Control para inspeccionar la atención de situaciones de emergencia declaradas, actividades donde se actúa como observadores de los procesos para identificar posibles riesgos que afecten la transparencia, probidad o el cumplimiento normativo, y alertar a los funcionarios sobre posibles desviaciones en la ejecución.

El control de las inversiones de emergencia social en las zonas de extrema pobreza se realizará preferentemente en la modalidad de control preventivo e incidirá en las obras de infraestructura educativa y de salud, electrificación rural, agua potable, saneamiento básico y promoción agraria, donde se constatará una actuación proactiva del Sistema Nacional de Control, con recomendaciones de mejora oportunas para la gestión, que permitan cumplir con los objetivos y metas de los proyectos, la prestación efectiva de los servicios y lograr los impactos sobre la población objetivo.

En el marco de la Ley de Control Interno No 28716 y de las Normas de Control Interno aprobadas por Resolución de Contraloría No 320-2006-CG, se promoverá la adecuada implementación del control interno establecido por la gerencia, vía tres mecanismos: a) incorporando en los programas de auditoría, procedimientos para evidenciar los problemas que afectan su buen funcionamiento, la determinación de altos riesgos en la entidad y la existencia de mecanismos de cautela para preservar el patrimonio público; b) evaluaciones del Órgano de Control Institucional sobre el comportamiento del sistema de control interno, cuyos resultados permitirán a la Contraloría General informar a la Comisión de Fiscalización y Contraloría; y, c) asistencia a la gerencia para mejorar los sistemas de 
control interno, principalmente en las áreas de gestión presupuestal, logística, personal, obras públicas y sistemas de información, entre otros.

Las acciones de control se orientan hacia las áreas sensibles a la corrupción, tales como la adquisición de bienes y servicios, contratación de obras públicas, inversión, personal y remuneraciones, otorgamiento de licencias y autorizaciones. Son igualmente prioritarios los procesos de privatización de empresas públicas y el otorgamiento de concesiones, donde se examinarán el uso y destino de los fondos provenientes de dichos ingresos.

Complementariamente, las acciones de control deben considerar el examen de los resultados de gestión, centrándose en el logro de objetivos y metas, y en las áreas vinculadas con la finalidad de las entidades. Tendrán prioridad las acciones dirigidas a los programas sociales de lucha contra la pobreza y a la provisión de los servicios básicos, donde se examinará el uso y destino de los recursos, el cumplimiento de los objetivos de los programas y metas de atención, así como la calidad del servicio provisto.

Priorizar la auditoría a los estados financieros y el examen especial a la información presupuestal de las entidades sujetas a control, con sujeción a la Ley de Gestión Presupuestaria del Estado y a las normas específicas dictadas por la Contraloría General.

El control presupuestal se orientará de modo preventivo al seguimiento del gasto efectuado con el propósito de alertar sobre posibles desviaciones en la ejecución.

El control presupuestal posterior, evaluará el cumplimiento de metas físico - financieras e informará sobre las medidas implementadas por el titular de la Entidad en cumplimiento de las normas de austeridad y racionalidad en el gasto público. La evaluación del cumplimiento de metas debe exami- nar la consistencia de las metas presupuestarias, sus productos y unidades de medida planificadas, en relación a los objetivos institucionales; siendo áreas de riego la unidad de planificación y presupuesto, y las áreas de línea vinculadas a la producción de los servicios. Respecto al cumplimiento de medidas de austeridad, se examinarán los gastos por la prestación de servicios públicos, la contratación de servicios no personales y las adquisiciones de bienes y servicios en general; así como las medidas adoptadas para restringir el gasto en materia de personal, servicios generales, viáticos, vehículos y movilidad, entre otros.

El control presupuestal en los Gobiernos Regionales y Locales dará especial atención a aquellos que reciben recursos provenientes del canon minero y petrolero, examinando su uso en proyectos u obras de impacto regional y local, conforme lo establece la normativa vigente; y el manejo de una cuenta exclusiva destinada para tales fines.

Las actividades de control se orientan a verificar el cumplimientos de actos de probidad y transparencia establecidos en las normas vigentes, tales como la presentación de declaraciones juradas de ingresos, bienes y de rentas; la publicación de información y atención de solicitudes relativas a la Ley de Transparencia y Acceso a la Información Pública, la actualización y difusión del TUPA y, en general, las rendiciones de cuenta a que están obligados los servidores del Estado.

La presentación de declaraciones juradas alcanza a los funcionarios públicos al tomar posesión de sus cargos, durante su ejercicio y al cesar en los mismos, conforme al artículo $42^{\circ}$ de la Constitución Política y las Leyes No 27482 y No 27785 ; la supervisión de la publicación de documentos legales en los portales institucionales se realiza de conformidad con la Ley No 29091 y su reglamento. 


\section{B. LINEAMIENTOS PARA EL CONTROL DE PROCESOS SENSIBLES A LA CORRUPCIÓN}

Control del Proceso de Adquisición de Bienes y Servicios, y Contratación de Obras

El control de las adquisiciones de bienes y servicios se realizará con un enfoque integral que comprenda la programación de los requerimientos, evaluación de propuestas, adjudicación, contratación y conformidad de la cantidad y calidad de los suministros; examinando la transparencia del proceso y publicación de los planes anuales de adquisición. Cuando corresponda, se verificará que los productos y servicios adquiridos sean de reducido impacto ambiental, no perjudicial para la salud y susceptible de reaprovechamiento. En la contratación de obras se verificará la existencia previa de los expedientes técnicos aprobados y los indicios de fraude en la adjudicación, negociación y ejecución del contrato.

Se dará prioridad al control de las exoneraciones de licitación pública por declaración de estados de urgencia, a los procesos de licitación con significación económica, a las adjudicaciones directas otorgadas por servicios personalísimos y a la contratación de servicios no personales.

Los programas de control incluirán procedimientos para revelar prácticas de fraccionamiento de bienes u obras, sobrevaluación de precios o presupuestos, evaluación indebida de postores, incumplimiento de contratos, omisión en el cobro de penalidades y adulteraciones en la cantidad y calidad de los bienes y servicios provistos.

En las entidades donde se ha producido procesos de modernización y cambios tecnológicos se examinarán las adquisiciones de equipos, hardware y sistemas de comunicación; la compra y/o desarrollo de software especializado y la implementación de los sistemas de gestión, intra e interconexión, evaluando la legalidad de estos procesos y los resultados e impactos sobre la mejora de la gestión.

Control del Proceso de Inversión

El control de proyectos de inversión pública examinará el cumplimiento de las metas físicas y financieras, centrándose en la eficiencia del uso de los recursos, donde se verificará si los precios contratados se ciñeron a los costos del expediente técnico o estudio de preinversión aprobado; si los pagos liquidados en la ejecución se ajustaron a los costos del expediente técnico y de pre inversión; y si se cumplieron con las metas totales del proyecto en los plazos previstos en los estudios. Se dará prioridad al control de proyectos con significación económica y social.

El control de la gerencia de proyectos incidirá en la observancia de los procedimientos establecidos en la Ley No 27293, del Sistema Nacional de Inversión Pública, su reglamento y normas complementarias, verificando que los proyectos cuenten con estudios de preinversión aprobados y declarados viables como requisito previo a su inicio. En la ejecución se incidirá en el pago de valorizaciones, adelantos y liquidaciones; y en el reconocimiento de adicionales de obra y ampliaciones de plazo; mientras que en la supervisión de las obras, se verificará el cumplimiento de las especificaciones técnicas y materiales utilizados por los contratistas.

Tienen prioridad para el control, los sub sectores Transportes, Vivienda, y las Instituciones Públicas Descentralizadas o Proyectos Especiales que ejecutan obras de infraestructura económica y social.

Control de Procedimientos Administrativos

El control de los procedimientos administrativos se realizará por la modalidad de 
acción de control en los casos donde existan indicios de presuntos arreglos comerciales con el sector privado, tales como las autorizaciones para actividades productivas, licencias de funcionamiento de negocios, otorgamiento de títulos de propiedad, autorizaciones de servicio de transporte público y uso de vías, permisos de operación y otorgamiento de subsidios o exoneraciones; así como por la modalidad de actividad de control, cuando los recursos de queja interpuestos por los administrados se refieran a procedimientos funcionales de alcance administrativo, como la expedición de certificados de salud y sanidad, patentes, documentos de identidad, estado civil, partidas u otros.

Se incidirá en la fijación de tarifas, simplificación de requisitos y en la atención de los usuarios dentro de los plazos aprobados en el TUPA, incidiendo en la aplicación del silencio administrativo positivo.

Control de Procesos de Personal, Remuneraciones y Pensiones

El control de los procesos de personal examinará la contratación y pago de remuneraciones y pensiones, así como el cumplimiento de las normas sobre incompatibilidades de los funcionarios y servidores del Estado en el ejercicio de sus cargos. Es de interés para el control, el examen a la contratación y supervisión de asesores y consultores con cargo a los recursos del Fondo de Apoyo Gerencial al Sector Público, donde se examinará la naturaleza del servicio especializado y su correspondencia con funciones de carácter misional.

Se incidirá en la detección de prácticas ilícitas relacionadas con el pago irregular de remuneraciones, bonificaciones, dietas y subvenciones, incluyendo los bonos de productividad.

\section{METODOS Y RESULTADOS}

Generales: No tienen un impacto sobre la calidad de las aseveraciones en los estados contables, dado que no se relacionan con la información contable.

Específicos: Se relacionan con la información contable y por lo tanto con las aseveraciones de los saldos de los estados contables. Este tipo de controles están desde el origen de la información hasta los saldos finales.

Control Interno Contable: Se refiere fundamentalmente a los procedimientos que se establecen referidos a la actividad de contabilidad, tales como, clasificador de cuentas y contenido de las mismas, estados financieros, registros y submayores, documentos para captar la información, etcétera.

Control Interno Administrativo: También destinado a salvaguardar los recursos institucionales, son esencialmente los controles que se establecen y funcionan independientemente de la contabilidad.

\section{ANALÍSIS Y DISCUSIÓN}

El Informe COSO internacionalmente se considera hoy en día como un punto de referencia obligado cuando se tratan materias de control interno, tanto en la práctica de las empresas como en los niveles legislativos.

La autora del presente trabajo concuerda con el concepto anterior pues responde a los intereses de todas las partes, facilita la comunicación y unificación de las expectativas: Confiabilidad de la información; Eficiencia y eficacia de las operaciones; Cumplimiento de las leyes, reglamentos y políticas, establecidas y Control de los recursos, de todo tipo, a disposición de la entidad.

Vol. 19(36) 2011 | QVIPURAMAYOC / 47 


\section{CONCLUSIONES:}

El control interno es un instrumento eficaz para lograr la eficiencia y eficacia en el trabajo de las entidades.

El concepto de control interno y su utilidad para guiar las operaciones ha ido calando paulatinamente, integrándose en los procesos y en la cultura de las entidades y los órganos públicos. Es notorio que los jerarcas y los titulares subordinados han reconocido su responsabilidad por establecer, mantener, perfeccionar y evaluar el sistema de control interno, así como por emprender las medidas necesarias para garantizar razonablemente su efectivo funcionamiento.

El control interno ha sido reconocido como una herramienta para que la administración de todo tipo de organización, obtenga una seguridad razonable sobre el cumplimiento de los objetivos institucionales y esté en capacidad de informar sobre su gestión a las personas interesadas en ella. En la Administración Pública, esa gestión debe regirse por los principios constitucionales y legales vigentes; además, la rendición de cuentas correspondiente tiene como destinatario último a la ciudadanía considerada de manera integral, pues las instituciones se crean para satisfacer necesidades de la colectividad, la cual tiene el poder supremo en una sociedad democrática.

El concepto razonable está relacionado con el reconocimiento explicito de la existencia de limitaciones inherentes del control interno.

La extensión de los controles adoptados en una organización también está limitada por consideración de costo, por lo tanto no es factible establecer controles que proporcionen protección absoluta del fraude y el despilfarro, sino establecer los controles que garanticen una seguridad razonable desde el punto de vista de los costos.

\section{REFERENCIAS BIBLIGRÁFICAS:}

1. Berbia, P. (2008). Evaluacíon Eficaz del Sistema de Control Interno. EE.UU: The Institute of Internal Auditors.

2. Timothy, B. (2007). Auditoría Basada en Riesgos. Bogotá: ECOE Editores.

3. Álvarez Illanes, J. F. (2007) Auditoría Gubernamental Integral. Lima - Perú. Pacífico Editores.

4. Federación Internacional de Contadores - IFAC. (2004) Manual Internacional de Pronunciamientos de Auditoria y Aseguramiento. Lima. Editado por la Junta de Decanos de Contadores Públicos del Perú.

5. Contraloría General de la República. (1998) Manual de Auditoría Gubernamental (MAGU). Lima: Editora Perú.

6. Contraloría General de la República. (1998). Normas Técnicas de control interno para el Sector Público. Lima. Editora Perú.

7. Contreras, E. (1995) Manual del Auditor. Lima: CONCYTEC

8. Porter, M. E. (1996) Estrategia Competitiva. México. Compañía Editorial Continental SA. De CV.

9. Porter, M. E. (1996) Ventaja competitiva. México. Compañía Editorial Continental SA. De CV.

10. Rodríguez Serpas, J. E. (2005) La auditoría Integral como alternativa para agregar valor a los servicios que presta el Contador Público. Bogotá. Editorial Norma.

11. Tuesta Riquelme, Y. (2000). "El ABC de la Auditoria Gubernamental”. Lima. Iberoamericana de Editores SA. 